\title{
SMOOTH MAPS, PULLBACK PATH SPACES, CONNECTIONS, AND TORSIONS
}

\author{
KUO-TSAI CHEN
}

\begin{abstract}
By generalizing the local version of the usual differential geometric notion of connections and that of torsions, a model for the pullback path space of a smooth map is constructed from the induced map of the de Rham complexes. The pullback path space serves not only as a homotopy fiber but also as a device reflecting differentiable properties of the smooth map. Applications are discussed.
\end{abstract}

Let $M$ and $N$ be $C^{\infty}$ manifolds or, more generally, differentiable spaces. Let $M$ be connected with a base point $x_{1}$ and let $P\left(M ;-, x_{1}\right)$ denote the space of smooth paths of $M$ ending at $x_{1}$. In order to study a smooth map $f: N \rightarrow M$, we associate functorially to $f$ the pullback space $E_{f}$ of the path fibration $P\left(M ;-, x_{1}\right) \rightarrow M$ through the map $f$. In a heuristic sense, the path space of manifold expresses its dynamics, and the pullback path space $E_{f}$ reflects the dynamics arising from the smooth map $f$.

Let $k$ denote either the real or the complex number field. The behavior of the pullback space $E_{f}$ is investigated through an algebraic model $K[Y ;[X]]$ constructed, under the assumption of simply-connectedness of $M$, as follows:

(a) Let $X=\left\{X_{1}, X_{2}, \ldots\right\}$ be a basis of the graded reduced homology group $\tilde{H}_{*}(M ; k)$ with the degree suppressed by 1 . We choose a "Hodge type" direct sum decomposition of the de Rham complex $\Lambda(M)$ and determine uniquely both a differential $\partial$ for the graded free associative algebra $k[X]$ and a generalized connection $\omega$ for the product vector bundle $k[X] \times M \rightarrow M$. With the differential $\partial, k[X]$ becomes a d.g. (differential graded) algebra.

(b) Let $Y=\left\{Y_{1}, Y_{2}, \ldots\right\}$ be a basis for $H_{*}(N ; k)$, and let $k[Y ;[X]]$ denote the free $k[X]$-module freely generated by $Y$. The de Rham complex $\Lambda(N)$ is a $\Lambda(M)$-module via the induced map $f^{*}: \Lambda(M) \rightarrow \Lambda(N)$. Again, we choose a "Hodge type" direct sum decomposition for $\Lambda(N)$ and determine uniquely both a generalized torsion $\Phi$ and a differential $\partial$ for $k[Y ;[X]]$, which now become a d.g. right $k[X]$-module.

It turns out that, under mild conditions, the algebraic model $(k[Y ;[X]], \partial)$ provides the correct cohomology. Moreover, the connection $\omega$ and the generalized torsion $\Phi$ give rise, through integration of differential forms, to a generalized

Received by the editors January 31, 1985 and, in revised form, November 18, 1985.

1980 Mathematic:s Subject Classification (1985 Revision). Primary 58A99: Secondary 53C99.

This work was supported in part by NSF Grant MCS 8200775.

1986 American Mathematical Society ()())2-9947/86\$1.00+\$.25 per page 
transport, namely, a chain map

$$
C_{*}\left(E_{f}\right) \rightarrow k[Y ;[X]]
$$

from the smooth chain complex $C_{*}\left(E_{f}\right)$ to the algebraic model with an induced homology isomorphism.

The mechanism of determining the differential $\partial$ of the d.g. algebra $k[X]$ via the de Rham complex $\Lambda(M)$ consists of the connection $\omega$ and a twisting cochain condition (see [5]). The mechanism of determining the differential $\partial$ and the transport for the d.g. right $k[X]$-module $k[Y ;[X]]$ via the map $f^{*}: \Lambda(M) \rightarrow \Lambda(N)$ consists of a generalized torsion $\Phi$ (called a canonical element) and a torsion condition. In $\$ \$ 1-4$, we explain the notions of connections, torsions, and transport and how the twisting cochain condition and the torsion condition yield both the differential and the transport (0.1).

A proof of Theorem 4.1 is given in $\$ 5$. In $\S 6$, we establish the isomorphism $H_{*}\left(E_{f} ; k\right) \simeq H_{*}(k[Y ;[X]] ; k)$ in the case where both $M$ and $N$ are $C^{\infty}$ manifolds with $M$ simply connected.

This work is a continuation of previous works on the special case where $N$ consists of a single point. This means that $E_{f}$ becomes the smooth loop space of $M$ and that the model is the noncommutative formal power series algebra $k[[X]]$ (see $[4,5]$ and Gugenheim [9]). In this case, Hain [11] gives an explicit relation between the model $(k[[X]], \partial)$ and Sullivan's minimal model.

From a pure homological-algebraic point of view, our model represents a "concise" Eilenberg-Moore spectral sequence in a one-sided case. It is obtained by a perturbation of the differential of the $E^{1}$ term. The reader is referred to the recent work [18] of Tanré regarding other models of similar nature (see also [1, 8, 10, $12,-14,16,17,19])$. A distinctive feature of our approach here is the transport $(0.1)$, which provides a direct link between the geometric and algebraic aspects of the method.

As in the author's previous works, the symbol $\Lambda$ is used to denote the de Rham complex functor.

1. Formal power series connections. Let $M$ be a $C^{\infty}$ manifold (or, more generally, a differentiable space in the sense of [5]).

Let $k$ be either the real or the complex number field. Let $k[[X]]$ be the algebra of formal power series in the indeterminates $X=\left\{X_{1}, X_{2}, \ldots\right\}$, which are noncommutative. Each $X_{i}$ is assigned a nonnegative degree $\operatorname{deg} X_{i}$ so that $k[[X]]$ is nonnegative graded.

Let $\Lambda(M)$ denote the de Rham complex of $C^{\infty}$ forms of $M$. Let $J$ be the automorphism of $\Lambda(M)$ given by $w \mapsto(-1)^{p} w$ for every $p$-form $w$ on $M$. Let $A$ be a differential graded subalgebra of $\Lambda(M)$. Let $A[[X]]$ be the noncommutative formal power series ring of the indeterminates $X$ with coefficients in $A$. Then the exterior differential $d$ and the operator $J$ extend from $A$ to $A[[X]]$ by acting on the coefficients. By a $k[[X]]$-valued connection on $M$ (restricted to $A$ ), we mean an element of $A[[X]]$ of the type

$$
\omega=\sum w_{i} X_{i}+\sum w_{i j} X_{i} X_{j}+\cdots,
$$


where the coefficients are forms belonging to $A$ with

$$
\operatorname{deg} w_{i}=1+\operatorname{deg} X_{i}, \quad \operatorname{deg} w_{i j}=1+\operatorname{deg} X_{i} X_{j}, \ldots
$$

2. The transport. Associated to every $k[[X]]$-valued connection $\omega$ on $M$, there is a transport

$$
T=1+\int \omega+\int \omega \omega+\cdots
$$

which is an element of $\Lambda(P(M))[[X]]$. For further details, see [4 and 5].

By a derivation $\partial$ of $k[[X]]$, we mean a graded linear endomorphism of degree -1 of $k[[X]]$ such that

$$
\partial\left(X_{i} X_{j} \cdots X_{k}\right)=\left(\partial X_{i}\right) X_{j} \cdots X_{j}+(-1)^{\operatorname{deg} X_{i}} X_{i} \partial\left(X_{j} \cdots X_{k}\right)
$$

and

$$
\partial\left(a_{0}+\sum a_{i} X_{i}+\sum a_{i j} X_{i} X_{j}+\cdots\right)=\sum a_{i} \partial X_{i}+\sum a_{i j} \partial\left(X_{i} X_{j}\right)+\cdots .
$$

Each derivation of $k[[X]]$ extends to a derivation of $\Lambda(M)[[X]]$ (or $\Lambda(P(M))[[X]])$.

Let $p_{0}, p_{1}: P(M) \rightarrow M$ be the endpoint maps such that $p_{0}(\gamma)=\gamma(0)$ and $p_{1}(\gamma)=\gamma(1)$. Thus $p_{0}^{*} \omega$ and $p_{1}^{*} \omega$ are elements of $\Lambda(P(M))[[X]]$. Theorem 3.3.1 of [5] states:

Let $\omega$ be a $k[[X]]$-valued connection on $M$. If $\partial$ is a derivation of $k[[X]]$ satisfying the twisting cochain condition

$$
\partial \omega+d \omega-J \omega \wedge \omega=0,
$$

then

$$
d T=\partial T-p_{0}^{*} \omega \wedge T+J T \wedge p_{1}^{*} \omega .
$$

EXAmple. Let $w$ be an $n$-form on $S^{n}$ so that $\int_{S^{n}} w=1, n>1$. Let $X$ be an indeterminate of degree $n-1$. Take $\omega=w X$ as a $k[[X]]$-valued connection on $S^{n}$. We have the transport

$$
T=1+\int w X+\int w w X^{2}+\cdots,
$$

where $\int w, \int w w, \ldots$ are forms on the path space $P(M)$ of respective degrees $n-1$, $2(n-1), \ldots$

Let $C_{*}(P(M))$ denote the normalized smooth cubic chain complex of $P(M)$. Then there is a map

$$
C_{*}\left(P\left(S^{n}\right)\right) \rightarrow k[[X]]
$$

through integration of the coefficients of $T$.

Let us equip $k[[X]]$ with the trivial differential $\partial$ so that $\partial\left(X_{i} X_{j} \cdots X_{k}\right)=0$. Then the twisting cochain condition holds. We have $d T=-p_{0}^{*} \omega \wedge T+J T \wedge p_{1}^{*} \omega$. The endpoint maps $p_{0}$ and $p_{1}$ restricted to the smooth loop space $P\left(S^{n} ; x_{1}, x_{1}\right)$ at a base point $x_{1}$ are constant maps. Therefore, restricted to the loop space $P\left(S^{n} ; x_{1}, x_{1}\right)$, we have $d T=0$. This leads to the conclusion that the map (2.4) restricted to $C_{*}\left(P\left(S^{n} ; x_{1}, x_{1}\right)\right)$ is a chain map. It turns out that this chain map induces a homology isomorphism $H_{*}\left(P\left(S^{n} ; x_{1}, x_{1}\right) ; k\right) \simeq k[[X]]$. 
3. The torsion condition. Denote by $k[Y ;[X]]$ the free graded right $k[[X]]$-module on the generators $Y=\left\{Y_{1}, Y_{2}, \ldots\right\}$ with $\operatorname{deg} Y_{\lambda} \geqslant 0$. A typical element of $k[Y ;[X]]$ can be written as

$$
\sum_{\lambda} Y_{\lambda}\left(a_{\lambda}+\sum a_{\lambda i} X_{i}+\sum a_{\lambda i j} X_{i} X_{j}+\cdots\right)
$$

with the $a$ 's belonging to $k$. A derivation $\partial$ of $k[[X]]$ can be extended to a derivation $\partial$ of $k[Y ;[X]]$ of degree -1 by assigning values to each $\partial Y_{\lambda}$.

Let $N$ be a $C^{\infty}$ manifold (or, more generally, a differentiable space), and let $f$ : $N \rightarrow M$ be a $C^{\infty}$ map. Let $B$ be a subcomplex of the de Rham complex $\Lambda(N)$ such that (a) the inclusion induces an isomorphism $H^{*}(B) \simeq H_{\mathrm{DR}}(N)$ and (b) $B$ is an $A$-module via $f^{*}$. Then $B[Y ;[X]]$ is well defined and is a right $A[[X]]$-module. A typical element of $B[Y ;[X]]$ can be written as

$$
\Phi=\sum_{\lambda} Y_{\lambda}\left(v_{\lambda}+\sum v_{\lambda i} X_{i}+\cdots\right), \quad v_{\lambda}, v_{\lambda i}, \ldots \in B
$$

Let $\omega$ be a $k[[X]]$-valued connection and $\partial$ a derivation of $k[[X]]$ so that the twisting cochain condition (2.2) holds. Let $\partial$ be extended to a derivation of $k[Y ;[X]]$, and let

$$
\Phi=\sum_{\lambda} Y_{\lambda}\left(v_{\lambda}+\sum v_{\lambda i} X_{i}+\cdots\right)
$$

be an element of $B[Y ;[X]]$ such that $\operatorname{deg} v_{\lambda i j \cdots k}=\operatorname{deg} Y_{\lambda} X_{i} X_{j} \cdots X_{k}$. We say that the pair $(\Phi, \partial)$ satisfies the torsion condition (with respect to the map $f$ and the connection $\omega)$ if

$$
\partial \Phi=d \Phi-J \Phi \wedge f^{*} \omega .
$$

Observe that the r.h.s. can be taken as the torsion of $\Phi$.

Let $x_{1}$ be a point (or a finite set of points) of $M$. Denote by $P\left(M ;-, x_{1}\right)$ the space of $C^{\infty}$ paths ending at $x_{1}$ in $M$. Then $P\left(M ;-, x_{1}\right)$ is a differentiable subspace of $P(M)$.

In order to see the role played by the torsion condition, we need to introduce the space $E_{f}=\left\{(y, \gamma): y \in N, \gamma \in P\left(M ;-, x_{1}\right), \gamma(0)=y\right\}$. In other words, $E_{f}$ is the pullback space in the pullback diagram

$$
\begin{array}{ccc}
E_{f} & \stackrel{\tilde{f}}{\rightarrow} & P\left(M ;-, x_{1}\right) \\
\downarrow \pi_{f} & & \downarrow p_{0} \\
N & \stackrel{f}{\rightarrow} & M
\end{array}
$$

where $\pi_{f}(y, \gamma)=y, \tilde{f}(y, \gamma)=\gamma$, and $p_{0}(\gamma)=\gamma(0)$. The space $E_{f}$ is known as the homotopy fiber of $f$, for $E_{f}$ is of the same homotopy type as the fiber in the case of a fibration. Now the transport $T$ is a formal power series in $X$ with coefficients being forms on $P(M)$. In this section, we shall restrict the coefficients of $T$ to the space $P\left(M ;-, x_{1}\right)$ so that $T$ shall be taken as an element of $\Lambda\left(P\left(M ;-, x_{1}\right)\right)[[X]]$. Then $\pi_{f}^{*} \Phi \wedge \tilde{f}^{*} T$ is a well-defined element of $\Lambda\left(E_{f}\right)[Y ;[X]]$ and gives rise to a 
linear map

$$
C_{*}\left(E_{f}\right) \rightarrow k[Y ;[X]]
$$

so that, for every smooth cube $\sigma$ of $E_{f}, \sigma \mapsto \int_{\sigma} \pi_{f}^{*} \Phi \wedge \tilde{f}^{*} T$.

Let $\omega$ be a $k[[X]]$-valued connection, and $\partial$ a derivation of $k[[X]]$ so that the twisting cochain condition (2.2) holds. Let the derivation $\partial$ be further extended to a derivation of $k[Y ;[X]]$. If the condition

$$
\partial\left(\pi_{f}^{*} \Phi \wedge \tilde{f}^{*} T\right)=d\left(\pi_{f}^{*} \Phi \wedge \tilde{f}^{*} T\right)
$$

holds, then the Stokes formula implies that the boundary operator of the normalized smooth cubic chain complex $C_{*}\left(E_{f}\right)$ and the derivation $\partial$ of $k[Y ;[X]]$ are compatible with the map (3.3). If $\partial \partial=0$, then (3.3) is a chain map.

LEMMA 3.1. If the pair ( $\Phi, \partial)$ satisfies the torsion condition (3.1), then the condition (3.4) holds.

Proof. The restriction of the endpoint map $p_{1}$ to $P\left(M ;-, x_{1}\right)$ is a constant map so that $p_{1}^{*} \omega=0$. The formula (2.3) becomes

$$
d T=\partial T-p_{0}^{*} \omega \wedge T .
$$

Using also the twisting cochain condition (2.2), we have

$$
\begin{aligned}
\partial\left(\pi_{f}^{*} \Phi \wedge \tilde{f}^{*} T\right) & =\pi_{f}^{*} \partial \Phi \wedge \tilde{f}^{*} T+\pi_{f}^{*}(J \Phi) \wedge \tilde{f}^{*} \partial T \\
& =\pi_{f}^{*}\left(d \Phi-J \Phi \wedge f^{*} \omega\right) \wedge \tilde{f}^{*} T+\pi_{f}^{*}(J \Phi) \wedge \tilde{f}^{*}\left(d T+p_{0}^{*} \omega \wedge T\right) .
\end{aligned}
$$

Hence (3.4) follows from the commutativity $f \pi_{f}=p_{0} \tilde{f}$.

4. The canonical element. The main objective of this paper is to study a $C^{\infty}$ map $f: N \rightarrow M$. Just for the sake of simplicity, let us assume that the homology groups of $M$ and $N$ over $k$ are finite dimensional. Related to this map $f$, a few constructions are given as follows:

(I) Let $\left[z_{1}\right], \ldots,\left[z_{m}\right]$ be a basis for the reduced homology group $\tilde{H}_{*}(M ; k)$. Let $X=\left\{X_{1}, \ldots, X_{m}\right\}$ be indeterminates so $\operatorname{deg} X_{i}=-1+\operatorname{deg}\left[z_{i}\right]$. Thus $k[[X]]$ can be taken as the completion of the graded tensor algebra on the graded vector space $V=s^{-1} \tilde{H}_{*}(M ; k)$ obtained from $\tilde{H}_{*}(M ; k)$ through depressing the degree by 1 .

(II) Let $Y=\left\{Y_{1}, \ldots, Y_{l}\right\}$ be a basis of $H_{*}(N ; k)$. Then we have $k[Y$; $[X]]$, which is the free right $k[[X]]$-module generated by $H_{*}(N ; k)$.

(III) Let $A$ be a differential graded subalgebra of $\Lambda(M)$ and $B$ a subcomplex of $\Lambda(N)$ such that the inclusions $A \subset \Lambda(M)$ and $B \subset \Lambda(N)$ respectively induce isomorphisms in cohomology. Assume, moreover, $B \wedge f^{*} A \subset B$.

(IV) We choose a direct sum decomposition.

$$
A=H_{A} \oplus C_{A} \oplus d A
$$

into graded subspaces, where $H_{A}$ consists of closed elements such that the inclusion $H_{A} \subset A$ induces an isomorphism $H_{A} \simeq H^{*}(A)$. It follows that $C_{A}$ consists of nonclosed elements and $d C_{A}=d A$. Similarly, we choose a direct sum decomposition

$$
B=H_{B} \oplus C_{B} \oplus d B \text {. }
$$


The decompositions (4.1A) and (4.1B) will be referred to as the respective Hodge type decompositions of $A$ and $B$.

Theorem 1.3.1 of [4] asserts that, with respect to every Hodge type decomposition (4.1A), there exists a unique pair $(\omega, \partial)$ satisfying the following conditions:

(a) $\omega=\sum w_{i} X_{i}+\sum w_{i j} X_{i} X_{j}+\cdots$ is a $k[[X]]-$ valued connection on $M$ restricted to $A$ such that $w_{1}, \ldots, w_{m} \in H_{A}$ and their cohomology classes $\left[w_{1}\right], \ldots,\left[w_{m}\right]$ form a basis dual to the homology basis $\left\{\left[z_{1}\right], \ldots,\left[z_{m}\right]\right\}$. Moreover, $w_{i j}, w_{i j k}, \ldots \in C_{A}$.

(b) $\partial$ is a derivation of $k[[X]]$ such that the twisting cochain condition (2.2) holds and $\partial \partial=0$.

Remark. Though Theorem 1.3.1 of [4] is stated for the case of $A=\Lambda(M)$, its proof is valid for any $A$ as described above.

THEOREM 4.1. Given the constructions (I)-(IV) as above, there exists a unique pair $(\Phi, \partial)$, where $\partial$ is a derivation of $k[Y ;[X]]$ compatible with that of $k[[X]]$, and

$$
\Phi=\sum_{\lambda} Y_{\lambda}\left(v_{\lambda}+\sum_{i} v_{\lambda i} X_{i}+\sum_{i, j} v_{\lambda i j} X_{i} X_{j}+\cdots\right)
$$

is an element of $B[Y ;[X]]$ satisfying the following conditions:

(a) $v_{\lambda} \in H_{B}$ and $v_{\lambda i}, v_{\lambda i j}, \ldots \in C_{B}$ with

$$
\operatorname{deg} v_{\lambda i j \cdots k}=\operatorname{deg}\left(Y_{\lambda} X_{i} X_{j} \cdots X_{k}\right) \text {. }
$$

(b) $\left\{\left[v_{1}\right], \ldots,\left[v_{l}\right]\right\}$ is a basis of $H_{\mathrm{DR}}^{*}(N)$ dual to the basis $\left\{Y_{1}, \ldots, Y_{l}\right\}$ of $H_{*}(N ; k)$.

(c) The pair $(\Phi, \partial)$ satisfies the torsion condition (3.1). Moreover $\partial \partial=0$.

We shall call $(\Phi, \partial)$ the canonical pair and $\Phi$ the canonical element of the $C^{\infty}$ map $f: N \rightarrow M$ with respect to the Hodge type decompositions (4.1A) and (4.1B).

The topological significance of the differential $\partial$ is that, under reasonable conditions, $H(k[Y ;[X]], \partial)$ coincides with $H_{*}\left(E_{f} ; k\right)$ provided $M$ is simply connected. More precisely, the complex $(k[Y ;[X]], \partial)$ has a natural filtration and gives rise to a spectral sequence, which is the Eilenberg-Moore spectral sequence. For details, see $\S 8$.

The next two examples seem to indicate that the canonical element $\Phi$ may have analytic significance.

EXAMPLE 1. Let $M$ and $N$ be compact Riemannian manifolds. Write $A=\Lambda(M)$ and $B=\Lambda(N)$. There are canonical Hodge decompositions of $A$ and $B$ such that $H_{A}$ and $H_{B}$ are the respective spaces of harmonic forms on $M$ and $N$. For every $C^{\infty}$ map $f: N \rightarrow M$, there is a unique pair $\left(\Phi_{f}, \delta_{f}\right)$. The canonical element

$$
\Phi_{f}=\sum Y_{\lambda}\left(v_{\lambda}(f)+\sum v_{\lambda i}(f) X_{i}+\cdots\right)
$$

is an invariant of the mapping space $C^{\infty}(M, N)$.

EXAMPLE 2. Let us consider a $C^{\infty}$ map

$$
f: N=R^{m} \rightarrow S^{n},
$$

where $S^{n}$ is taken as a Riemannian manifold.

According to step (I), we have a single indeterminate $X$ of degree $n-1$. In step (II), $H_{*}\left(R^{m}: k\right)$ is generated by a single element $Y$ of degree 0 . In step (III), let $A=\Lambda\left(S^{m}\right)$ and $B=\Lambda\left(R^{m}\right)$. Take any Hodge type decompositions (4.1A) and 
(4.1B). Then $H_{B}=k$ and $H_{A}$ has a basis $\{1, w\}$ where $w$ is an $n$-form with $\int_{S^{\prime \prime}} w=1$. Let the pair $(\omega, \partial)$ be such that $\omega=w X$ and $\partial$ is a trivial differential. Then conditions of step (IV) hold. According to Theorem 4.1, there is a unique pair $(\Phi, \partial)$ with

$$
\Phi=Y\left(1+v_{1} X+v_{2} X^{2}+\cdots\right), \quad \operatorname{deg} V_{i}=i(n-1),
$$

so that $\partial \Phi=d \Phi-J \Phi \wedge f^{*} \omega$.

If we let $\partial$ be the trivial differential of $k[Y ;[X]]$ and let $v_{i} \in C_{B}$ be recursively determined by the condition

$$
d v_{i}=(-1)^{i(n-1)} v_{i-1} \wedge f^{*} w, \quad i \geqslant 1,
$$

then the pair $(\Phi, \partial)$ will meet the requirement and is uniquely determined.

Let $l$ be the largest integer such that $v_{l} \neq 0$. Then $l$ is an invariant of the $C^{\infty}$ map $f$, which depends only on the choice of the Riemannian metric for $S^{n}$ and that of the Hodge type decomposition for $\Lambda\left(R^{m}\right)$.

Finally we observe that the usual proof for the Poincare lemma for $R^{m}$ is based on a deformation $F: R^{m} \times I \rightarrow R^{m}$ given by $(x, t) \mapsto t x$. This induces a cochain homotopy $\int_{F}: B \rightarrow B$ of degree -1 such that, for every $p$-form $u$ on $R^{m}, p>0$, $u=\int_{F} d u+d \int_{F} u$. This means that, if the euclidean structure and the origin of $R^{m}$ are given, then there is a canonical Hodge type decomposition of $B$ with $H_{B}=k$ and $C_{B}=\int_{F} d B$.

5. Proof of Theorem 4.1. Let $\Psi \in B[Y ;[X]]$. Given $s \geqslant 0$, let $[\Psi]_{s}$ denote the sum of all terms of the type $Y_{\lambda} X_{i_{1}} \cdots X_{i_{s}}$ of $\Psi$, and set

$$
\Psi_{(s)}=[\Psi]_{0}+[\Psi]_{1}+\cdots+[\Psi]_{s}
$$

which is obtained from $\Psi$ by truncating terms of all $Y_{\lambda} X_{i_{1}} \cdots X_{i_{r}}$ with $r>s$.

According to Theorem 1.3.1 of [4], the Hodge type decomposition (4.1A) yields a unique pair $(\omega, \partial)$ as described before. In order to extend the derivation $\partial$ of the graded algebra $k[[X]]$ to that of $k[Y ;[X]]$ as a graded right $k[[X]]$-module, it suffices to specify values of each $\partial Y$.

We are going to inductively construct a sequence $\left(\Phi_{(s)}, \partial_{s}\right)$ on $s \geqslant 0$ satisfying the following conditions:

(a) $\Phi_{(s)}=\Phi_{(s-1)}+\sum v_{\lambda i_{1} \cdots i_{s}} Y_{\lambda} X_{i_{1}} \cdots X_{i_{s}}$ with $\Phi_{(-1)}=0$ where each $v_{\lambda i_{1} \cdots i_{s}}$ is a form on $N$ of degree equal to $\operatorname{deg}\left(Y_{\lambda} X_{i_{1}} \cdots X_{i_{s}}\right)$.

(b) $\partial_{s}$ is a derivation of $k[Y ;[X]]$ compatible with the derivation $\partial$ of $k[[X]]$ such that

$$
\partial_{s} Y_{\lambda}=\partial_{s-1} Y_{\lambda}+\sum b_{\mu i_{1} \cdots i_{s}}^{(\lambda)} X_{i_{1}} \cdots X_{i_{s}}
$$

with $\partial_{-1} Y_{\lambda}=0$ and the coefficients $b_{\mu i_{1} \cdots i_{s}}^{(\lambda)} \in k$.

(c) The condition

$$
\left(\partial_{s} \Phi_{(s)}-d \Phi_{(s)}+J \Phi_{(s)} \wedge f^{*} \omega\right)_{(s)}=0
$$

holds. 
We first observe that the above conditions imply

$$
\left(\partial_{s} \partial_{s} \Phi_{(s)}\right)_{(s+1)}=0 \text {. }
$$

In fact,

$$
\begin{aligned}
\left(\partial_{s} \partial_{s} \Phi_{(s)}\right)_{(s+1)} & =\left(\partial_{s}\left(d \Phi_{(s)}-J \Phi_{(s)} \wedge f^{*} \omega\right)\right)_{(s+1)} \\
& =\left(d\left(\partial_{s} \Phi_{(s)}\right)-J \partial_{s} \Phi_{(s)} \wedge f^{*} \omega-\Phi_{(s)} \wedge f^{*} \partial \omega\right)_{(s+1)} \\
& =\left(d\left(d \Phi_{(s)}-J \Phi_{(s)} \wedge f^{*} \omega\right)-J\left(d \Phi_{(s)}-J \Phi_{(s)} \wedge f^{*} \omega\right)\right. \\
& \left.\wedge f^{*} \omega-\Phi_{(s)}-\Phi_{(s)} \wedge f^{*}(-d \omega+J \omega \wedge \omega)\right)_{(s+1)} \\
& =0 .
\end{aligned}
$$

Let $\imath^{\prime s}$ denote the totality of elements $\Psi$ of $B[Y ;[X]]$ such that

$$
[\Psi]_{0}=[\Psi]_{1}=\cdots=[\Psi]_{s-1}=0
$$

It is clear that, for every element $\Psi \in \mathfrak{\Im}^{\prime 2}, \partial_{s} \Phi \equiv \partial_{s-1} \Psi \bmod \mathfrak{J}^{\prime+1}$.

Our problem is to construct, by induction on $s$, the forms $v_{\lambda i_{1} \cdots i_{s}}$ and the coefficients $b_{\mu i_{1} \cdots i}^{(\lambda)}$. Observe that $\Phi_{(0)}=\sum v_{\lambda} Y_{\lambda}$ and

$$
\partial_{s}\left(\Phi_{(s)}-\Phi_{(0)}\right)=\partial_{s-1}\left(\Phi_{(s-1)}-\Phi_{(0)}\right) \bmod \mathfrak{s}^{\prime s+1} .
$$

Moreover,

$$
\left(J \Phi_{(s)} \wedge f^{*} \omega\right)_{(s)}=\left(J \Phi_{(s-1)} \wedge f^{*} \omega\right)_{(s)}
$$

Thus (5.1) yields

$$
\sum\left(h_{\lambda i_{1} \cdots i_{v}}-d v_{\lambda i_{1} \cdots i_{s}}\right) Y_{\lambda} X_{i_{1}} \cdots X_{i_{s}}=R_{s}
$$

where $h_{\lambda i_{1} \cdots i_{1}}=\sum b_{\lambda i_{1} \cdots i_{s}}^{(\lambda)} v_{\mu}$ and

$$
R_{s}=\left(-\partial_{s-1} \Phi_{(s-1)}+d \Phi_{(s-1)}-J \Phi_{(s-1)} \wedge f * \omega\right)_{(s)} .
$$

It remains to solve the equation (5.3) $)_{s}$ for $h_{i_{1} \cdots i_{s}} \in H_{B}$ and $v_{\lambda i_{1} \cdots i_{s}} \in C_{B}$. According to the induction hypothesis, $R_{s}$ is known, and $R_{s} \equiv 0 \bmod \mathfrak{\Im}^{\prime s}$ so that

$$
R_{s}=\sum r_{\lambda i_{1} \cdots i_{1}} Y_{\lambda} X_{i_{1}} \cdots X_{i_{s}}
$$

Also,

$$
\begin{aligned}
d R_{s} \equiv & -\partial_{s-1} d \Phi_{(s-1)}+\left(J d \Phi_{(s-1)}\right) \wedge f^{*} \omega-\Phi_{(s-1)} \wedge f^{*} d \omega \bmod \mathfrak{\Im}^{s+1} \\
\equiv & -\partial_{s-1} d \Phi_{(s-1)}+\left(J \partial_{s-1} \Phi_{(s-1)}+\Phi_{(s-1)} \wedge J f^{*} \omega\right) \wedge f^{*} \omega \\
& -\Phi_{(s-1)} \wedge f^{*} d \omega \bmod \mathfrak{\Im}^{\prime s+1} \\
\equiv & -\partial_{s-1} d \Phi_{(s-1)}+\left(J \partial_{s-1} \Phi_{(s-1)}\right) \wedge f^{*} \omega+\Phi_{(s-1)} \wedge \partial f^{*} \omega \bmod \mathfrak{\Im}^{\prime s+1} \\
\equiv & -\partial_{s-1} d \Phi_{(s-1)}+\partial_{s-1}\left(J \Phi_{(s-1)} \wedge f^{*} \omega\right) \quad \bmod \mathfrak{\Im}^{\prime s+1} \\
\equiv & -\partial_{s-1} \partial_{s-1} \Phi_{(s-1)} \bmod \mathfrak{\Im}^{\prime s+1} \\
\equiv & 0 \bmod \mathfrak{J}^{\prime s+1} .
\end{aligned}
$$


This means that each form $r_{\lambda i_{1} \cdots i_{s}}$ is closed and belongs to $H_{B} \oplus d B=H_{B} \oplus d C_{B}$. Hence the theorem is proved.

6. Topological justification. This section contains a proof of the next result.

THEOREM 6.1. Let $M$ and $N$ be $C^{\infty}$ manifolds having finite Betti numbers, and let $M$ be simply connected. Then the chain map (3.3) induces an isomorphism

$$
H_{*}\left(E_{f} ; k\right) \simeq H_{*}(k[Y ;[X]]) \text {. }
$$

As in $\$ 4.2$ of [6], we use $A_{f}^{\prime}$ to denote the subcomplex of $\Lambda\left(E_{f}\right)$ spanned by all elements of the type $\pi_{f}^{*} v \wedge f^{*} \wedge \int w_{1}^{\prime} \cdots w_{r}^{\prime}$ with $v \in \Lambda(N)$ and $w_{1}^{\prime}, \ldots, w_{r}^{\prime} \in A$, $r \geqslant 0$. Let $\bar{B}(A)$ denote the reduced bar construction of $A$ (in the usual sense under the conditions: $A^{0}=k$ and $A^{1} \cap d \Lambda^{0}(M)=0$ and, otherwise, in a slightly modified sense as in [13]). Then there is an isomorphism

$$
\Lambda(N) \otimes \bar{B}(A) \simeq A_{f}^{\prime}
$$

such that $v \otimes\left[w_{1}^{\prime}|\cdots| w_{r}^{\prime}\right] \mapsto \pi_{f}^{*} v \wedge \tilde{f}^{*} \int w_{1}^{\prime} \cdots w_{r}^{\prime}$, where $\Lambda(N) \otimes \bar{B}(A)$ is just the one-sided bar construction $B(\Lambda(N)|A| k)$ with $\Lambda(N)$ taken as a differential graded $A$-module via the map $A \subset \Lambda(M) \stackrel{f^{*}}{\rightarrow} \Lambda(N)$. Theorem 0.1 of [6] (Theorem 4.3.1 of [5]) implies that there is an isomorphism $H^{*}\left(A_{f}^{\prime}\right) \simeq H^{*}\left(E_{f}, k\right)$ through integration.

The dual of the chain map (3.3) has a factorization

$$
\operatorname{Hom}_{k}(k[Y ;[X]], k) \stackrel{\tau}{\rightarrow} A_{f}^{\prime} \rightarrow \operatorname{Hom}_{k}\left(C_{*}\left(E_{f}\right), k\right)
$$

given by

$$
h \mapsto h\left(\pi_{f}^{*} \Phi \wedge \tilde{f}^{*} T\right) \mapsto \int h\left(\pi_{f}^{*} \Phi \wedge \tilde{f}^{*} T\right),
$$

where the linear functional $h$ on $k[Y ;[X]]$ is extended to $B[Y ;[X]]$, and the integral $\int h\left(\pi_{f}^{*} \Phi \wedge \tilde{f}^{*} T\right)$ is taken to be a smooth cochain. It follows from (3.4) that $\tau$ is a cochain map. In fact

$$
\tau \delta h=h\left(\partial\left(\pi_{f}^{*} \Phi \wedge \tilde{f}^{*} T\right)\right)=d \tau h .
$$

It remains to show that $\tau$ induces an isomorphism

$$
H^{*}(k[Y ;[X]]) \simeq H^{*}\left(A_{f}^{\prime}\right) .
$$

This will be verified through spectral sequences.

The chain complex $k[Y ;[X]]$, which may be taken as a subcomplex of $B[Y ;[X]]$, has a descending filtration $\left\{\mathfrak{\Im}^{r}\right\}$ with $\mathfrak{\Im}^{r}=k[Y ;[X]] \cap \mathfrak{\Im}^{\prime r}$. The resulting spectral sequence $\left\{\hat{E}^{r}\right\}$ is such that

$$
\hat{E}_{p}^{1}=H_{*}(N ; k) \otimes\left(\otimes P^{P^{-1}} \tilde{H}_{*}(M ; k)\right),
$$

where the graded group $s^{-1} \tilde{H}_{*}(M ; k)$ is obtained from $H_{*}(M ; k)$ by replacing $H_{0}(M ; k)$ with 0 and then depressing the degree by 1 . The dual cochain complex $\operatorname{Hom}_{k}(k[Y ;[X]], k)$ has an ascending filtration, whose resulting spectral sequence $\left\{\hat{E}_{r}\right\}$ is such that

$$
\hat{E}_{1}^{p}=H^{*}(N ; k) \otimes\left(\otimes \mathcal{P}^{-1} \tilde{H}^{*}(M ; k)\right),
$$

where the graded group $s^{-1} \tilde{H}^{*}(M ; k)$ is dual to $s^{-1} \tilde{H}_{*}(M ; k)$. 
The complex $A_{f}^{\prime} \approx \Lambda(N) \otimes \bar{B}(A)$ has an ascending filtration $\left\{\mathfrak{F}^{r} A_{f}^{\prime}\right\}$ whose resulting spectral sequence $\left\{E_{r}\right\}$ is such that

$$
E_{1}^{p}=H_{\mathrm{DR}}^{*}(N) \otimes\left(\otimes{ }^{p} S^{-1} H_{\mathrm{DR}}^{*}(M)\right) .
$$

Now we are going to verify that the map $\tau$ preserves filtration. Let $\left\{x_{\lambda i_{1} \cdots i_{s}}\right\}$ be the basis of the graded space $\operatorname{Hom}_{k}(k[Y ;[X]], k)$ dual to the basis $\left\{Y_{\lambda} X_{i_{1}} \cdots X_{i_{s}}\right\}$ of $k[Y ;[X]]$. Let $\left\{\mathfrak{\Im}_{r}^{*}\right\}$ be the filtration of $\operatorname{Hom}_{k}(k[Y ;[X]], k)$. Then $\mathfrak{\Im}_{r}^{*}$ has a basis consisting of all $x_{\lambda i_{1} \ldots i_{1}}, s>r$. Recall that

$$
\omega=\sum w_{i} X_{i}+\sum w_{i j} X_{i} X_{j}+\cdots
$$

and

$$
\begin{aligned}
T & =1+\int \omega+\int \omega \omega+\cdots \\
& =1+\sum \int w_{i} X_{i}+\sum\left(\int w_{i} w_{j}+\int w_{i j}\right) X_{i} X_{j}+\cdots
\end{aligned}
$$

We make the identification $A_{f}^{\prime}=\Lambda(N) \otimes \bar{B}(A)$ and write $v \otimes\left[w_{1}^{\prime}|\cdots| w_{r}^{\prime}\right]=$ $v\left[w_{1}^{\prime}|\cdots| w_{r}^{\prime}\right]$. Then

$$
\begin{aligned}
\pi_{f}^{*} \Phi \wedge \tilde{f}^{*} T= & \sum Y_{\lambda}\left(\pi_{f}^{*} v_{\lambda}+\sum \pi_{f}^{*} v_{\lambda i} X_{i}+\cdots\right) \wedge \tilde{f}^{*} T \\
= & \sum v_{\lambda}[\quad] Y_{\lambda}+\sum\left(v_{\lambda}\left[w_{i}\right]+v_{\lambda i}\right) Y_{\lambda} X_{i} \\
& +\sum\left(v_{\lambda}\left(\left[w_{i} \mid w_{j}\right]+\left[w_{i j}\right]\right)+v_{\lambda i}\left[w_{j}\right]+v_{\lambda i j}\right) Y_{\lambda} X_{i} X_{j}+\cdots .
\end{aligned}
$$

Since $\tilde{F}^{r} A_{f}^{\prime}$ is spanned by all $v\left[w_{1}^{\prime}|\cdots| w_{s}^{\prime}\right], s<r$, we have

$$
\tau\left(x_{\lambda}\right)=v_{\lambda}[\quad], \quad \tau\left(x_{\lambda i}\right)=v_{\lambda}\left[w_{i}\right]+v_{\lambda i}[] \equiv v_{\lambda}\left[w_{i}\right] \bmod \mathfrak{F}^{1} A_{f}^{\prime},
$$

and, in general,

$$
\tau\left(x_{\lambda i_{1} \ldots i_{r}}\right) \equiv v_{\lambda}\left[w_{i_{1}}|\cdots| w_{i_{r}}\right] \bmod \mathfrak{F}^{r} A_{f}^{\prime}
$$

Clearly $\tau$ preserves filtration and, moreover, induces an isomorphism $\hat{E}_{1} \simeq E_{1}$.

The spectral sequences $\left\{\hat{E}_{r}\right\}$ and $\left\{E_{r}\right\}$ converge respectively to $H^{*}(k[Y ;[X]])$ and $H^{*}\left(A_{f}^{\prime}\right)$. Hence we have the isomorphism (6.2), and the theorem is proved.

\section{BIBLIOGRAPHY}

1. D. Anick, $A$ model of Adam-Hilton type for fiber squares (preprint).

2. K. T. Chen, Connection, holonomy and path space homologv, Differential Geometry, Proc. Sympos. Pure Math., vol. 27, Amer. Math. Soc., Providence, R.I., 1975, pp. 39-52.

3. Reduced bar constructions on de Rham complexes, Collection of Papers in Honor of Samuel Eilenberg, Academic Press, New York, 1976, pp. 19-32.

4. Extension of $C^{\infty}$ function algebra by integrals and Malcev completion of $\pi_{1}$, Adv. in Math. 23 (1977), 181-210.

5. It Iterated path integrals, Bull. Amer. Math. Soc. 83 (1977), 831-879.

6. Pullback de Rham cohomology of the free path fibration, Trans. Amer. Math. Soc. 242 (1978), 307-318.

7. P. Deligne, P. Griffiths, J. Morgan and D. Sullivan, Real homotopy theory of Kähler manifolds, Invent. Math. 29 (1975), 245-274.

8. P. Grivel, Formes différentielles et suites spectrales, Ann. Inst. Fourier (Grenoble) 29 (1979), 17-37.

9. V. K. A. M. Gugenheim, On a modified Eilenherg-Moore Theorem, Geometric Applications of Homotopy Theory. II, Lecture Notes in Math., vol. 658, Springer-Verlag, Berlin, 1978, pp. 177-190. 
10. V. K. A. M. Gugenheim and J. P. May, On the theory and applications of differential torsion products, Mem. Amer. Math. Soc. No. 142 (1974).

11. R. M. Hain, Twisting cochains and duality between minimal algebras and minimal Lie algebras, Trans. Amer. Math. Soc. 277 (1983), 397-411.

12. S. Halperin, Rational fibrations, minimal models, and fibrings of homogeneous spaces, Trans. Amer. Math. Soc. 244 (1978), 199-222.

13. S. Halperin and J. Stasheff, Obstructions to homotopy equivalences, Adv. in Math. 32 (1979), 233-279.

14. J. M. Lamaire, Modeles minimaux pour les algebres de chaines, Publ. Dép. Math. (Lyon) 13 (1976), $13-26$.

15. D. Quillen, Rational homotopy theory, Ann. of Math. (2) 90 (1969), 205-295.

16. L. Smith, Lectures on the Eilenberg-Moore spectral sequence, Lecture Notes in Math., vol. 134, Springer-Verlag, Berlin and New York, 1970.

17. D. Sullivan, Infinitesimal computations in topology, Inst. Hautes Études Sci. Publ. Math. 47 (1977), 269-331.

18. D. Tanré, Homotopie rationelle: Modèles de Chen, Quillen, Sullivan, Lecture Notes in Math., vol. 1025, Springer-Verlag, Berlin and New York, 1983.

19. W. T. Wu, Theory of $I^{*}$-functors in algebraic topology, Sci. Sinica 18 (1975), 464-482.

Department of Mathematics, University of Illinois, Urbana, Illinois 61801 